\title{
Helicopter Flight Test of 3-D Imaging Flash LIDAR Technology for Safe, Autonomous, and Precise Planetary Landing
}

\author{
Vincent Roback ${ }^{1}$, Alexander Bulyshev $^{2}$, Farzin Amzajerdian $^{1}$, and Robert Reisse ${ }^{1}$ \\ 1. NASA Langley Research Center, Hampton, Virginia 23681, USA \\ 2. Analytical Mechanics Associates, Inc., Hampton, Virginia 23666, USA
}

\begin{abstract}
Two flash lidars, integrated from a number of cutting-edge components from industry and NASA, are lab characterized and flight tested for determination of maximum operational range under the Autonomous Landing and Hazard Avoidance Technology (ALHAT) project (in its fourth development and field test cycle) which is seeking to develop a guidance, navigation, and control (GN\&C) and sensing system based on lidar technology capable of enabling safe, precise crewed or robotic landings in challenging terrain on planetary bodies under any ambient lighting conditions. The flash lidars incorporate pioneering 3-D imaging cameras based on Indium-Gallium-Arsenide Avalanche Photo Diode (InGaAs APD) and novel micro-electronic technology for a $128 \times 128$ pixel array operating at $30 \mathrm{~Hz}$, high pulse-energy $1.06 \mu \mathrm{m} \mathrm{Nd}$ :YAG lasers, and high performance transmitter and receiver fixed and zoom optics. The two flash lidars are characterized on the NASA-Langley Research Center (LaRC) Sensor Test Range, integrated with other portions of the ALHAT GN\&C system from partner organizations into an instrument pod at NASA-JPL, integrated onto an Erickson Aircrane Helicopter at NASA-Dryden, and flight tested at the Edwards AFB Rogers dry lakebed over a field of humanmade geometric hazards during the summer of 2010. Results show that the maximum operational range goal of $1 \mathrm{~km}$ is met and exceeded up to a value of $1.2 \mathrm{~km}$. In addition, calibrated 3-D images of several hazards are acquired in realtime for later reconstruction into Digital Elevation Maps (DEM's).
\end{abstract}

Keywords: Flash Lidar, Laser Remote Sensing, 3-D Imaging, Characterization, Safe Landing, Hazard Detection, ALHAT, Flight Test, Planetary or Lunar Landing, Laser RADAR

\section{INTRODUCTION}

The imaging flash lidar is being considered as the primary sensor, due to its ability to provide three-dimensional (3-D) images of surfaces and hazards, for future robotic and crewed landing missions to planetary bodies including the moon, Mars, asteroids etc. Future human exploration missions will similarly require precise landing with increased levels of safety over those exercised in the Apollo program if they are to become more commonplace. Despite the successes of the Apollo program (which sought to land under favorable lighting conditions at sites without significant terrain challenges) two of the six missions experienced near disaster during the landing phase with all six landings being perilous ${ }^{1}$. An imaging lidar system records a 3-D image of a scene by converting intensity versus time of flight of short laser pulses into intensity versus distance along the line of sight for each spatially resolved area within a two-dimensional (2-D) image. In older, more conventional imaging lidar systems, each 2-D pixel is recorded with a separate laser pulse. Thus many laser pulses are required to record large, multi-pixel images. A flash lidar system records full 3-D images with a single laser pulse, permitting higher data rates and freezing out movement within the scene and motion of the transmitter/receiver platform. The need for high speed raster scanners to sequentially address image pixels is also eliminated. The receiver is much like the familiar digital camera, but with "smart pixels" that are capable of recording the required sequential temporal information. LaRC has been assessing the potentials of flash lidar technology as a landing sensor and working on its advancement under the ALHAT project for the past six years. The ALHAT project, led by NASA Johnson Space Center, is established by NASA to develop and demonstrate a guidance, navigation, and control system for future planetary landing missions capable of terrain hazard avoidance (see Figure 1) and precision landing under any lighting conditions anywhere on the body ${ }^{2}$. 


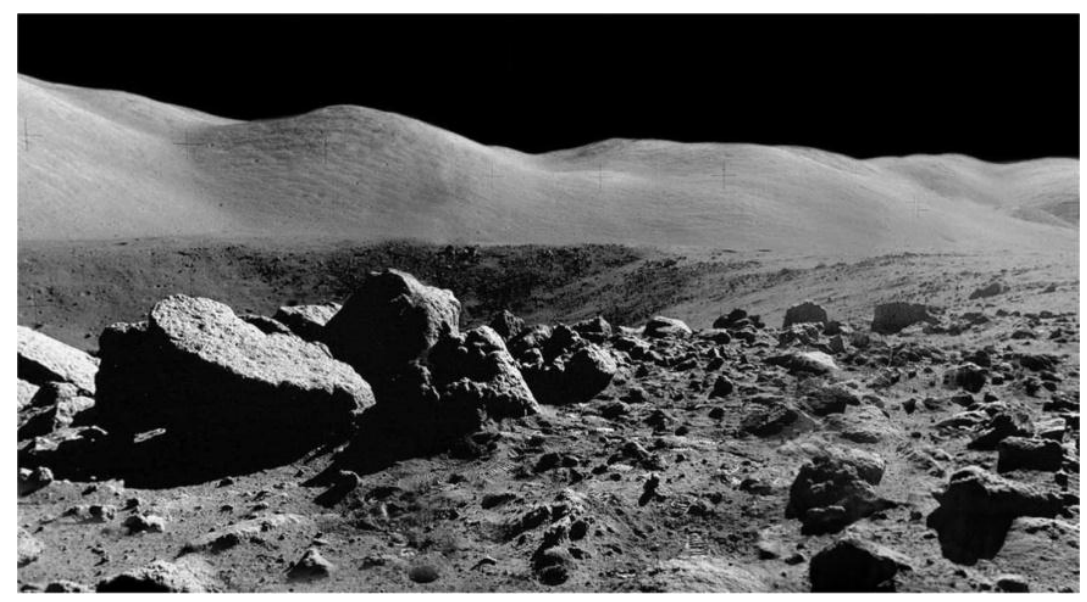

Figure 1: Future planetary landing missions (moon shown here) are considering scientifically interesting sites near craters and rough terrain.

To fulfill the requirements of global planetary body access under any lighting conditions, ALHAT is pursuing active sensor technology development and maturation to implement five sensor functions: altimetry, velocimetry, Terrain Relative Navigation (TRN), Hazard Detection and Avoidance (HDA) and Hazard Relative Navigation (HRN). Table 1 lists the ALHAT sensor suite with each sensor's top level performance specifications for achieving each of required functions with some degree of redundancy. The flash lidar is being considered for performing all three functions with the exception of velocimetry for which a Doppler Lidar is being developed. The ability of the Doppler Lidar to provide velocity data with approximately one centimeter / second is highly attractive for precision landing. Additionally, the Doppler Lidar provides high resolution altitude and ground-relative attitude data that may further improve precision navigation to the identified landing site. A description of the Doppler lidar and its capabilities have been reported earlier 3,4 . The Laser Altimeter provides independent altitude data over a large operational altitude range of $20 \mathrm{~km}$ to $100 \mathrm{~m}$. All three laser sensors have a nominal update rate of $30 \mathrm{~Hz}$.

Table 1: ALHAT Sensor Suite.

\begin{tabular}{|l|l|l|l|}
\hline Sensor & Function & Operational Altitude Range & Precision/Resolution \\
\hline \multirow{3}{*}{ Flash Lidar } & HDA/HRN & $1000 \mathrm{~m}-100 \mathrm{~m}$ & $5 \mathrm{~cm} / 40 \mathrm{~cm}$ \\
\cline { 2 - 4 } & TRN & $15 \mathrm{~km}-5 \mathrm{~km}$ & $20 \mathrm{~cm} / 6 \mathrm{~m}$ \\
\cline { 2 - 4 } & Altimetry & $20 \mathrm{~km}-100 \mathrm{~m}$ & $20 \mathrm{~cm}$ \\
\hline \multirow{2}{*}{ Doppler Lidar } & Velocimetry & $2500 \mathrm{~m}-10 \mathrm{~m}$ & $1 \mathrm{~cm} / \mathrm{sec}$ \\
\cline { 2 - 4 } & Altimetry & $2500 \mathrm{~m}-10 \mathrm{~m}$ & $5 \mathrm{~cm}$ \\
\hline Laser Altimeter & Altimetry & $20 \mathrm{~km}-100 \mathrm{~m}$ & $20 \mathrm{~cm}$ \\
\hline
\end{tabular}

All five of the aforementioned functions provide input to the navigation filter for landing vehicle state estimation, flight trajectory retargeting, and maneuvering to a safe site. Of these five functions, altimetry and velocimetry are direct sensor measurements, whereas the TRN, HDA, and HRN functions can be considered relative measurements, since the sensor output is derived from a correlation with either "a priori" terrain information, or with a sequence of previous sensor measurements. The latter functions can also be considered techniques, since a number of sensor / algorithm combinations can achieve similar results under the appropriate concept of operation. The location determination of safe landing sites is made from the location determination of hazards, simultaneously recorded within full 3-D images in complete spatial and temporal resolution. The simultaneity of the recording of full 3-D scenes with single laser pulses not only enables more rapid acquisition, but simpler and more rapid processing of scene information to enable the time-sensitive precision navigation necessary to avoid hazards and land precisely at the retargeted location. 


\section{FLASH LIDAR OPERATIONAL CONCEPT}

The Flash Lidar serves as a multi-functional sensor capable of performing four out of five ALHAT functions. The current operational concept (Figure 2) is based on a Flash Lidar system capable of generating 256x256 pixel image frames from nominally a one kilometer distance. The Flash Lidar initiates its operation in an altimetry mode after the vehicle begins its breaking phase and its altitude drops to approximately $20 \mathrm{~km}$ above the ground. In the altimetry mode of operation, the lidar transmitter laser is focused such that only a few of the detector array pixels are illuminated. The focusing of the laser increases the operational range of the lidar from one kilometer (with all of its pixels illuminated) to $20 \mathrm{~km}$ (with only about 100 illuminated pixels). The altitude data is then provided to the flight computer to update the inertial measurement unit (IMU) data and improve the vehicle position estimate from more than $1 \mathrm{~km}$ to about $300 \mathrm{~m}$.

At approximately $15 \mathrm{~km}$ above ground level, the flash lidar will switch to the TRN (which has been previously reported ${ }^{5}$ ) mode by slightly increasing the transmitter beam divergence to illuminate approximately 200 pixels. In this mode, the Flash Lidar will continue to provide altitude data as well as generating consecutive 3-D images of the terrain with a lateral resolution of less than six meters which is sufficient for performing the TRN function. The acquired images are correlated with stored on-board reference maps of known features such as craters and surface elevation data. The correlations are used to estimate the relative position error (map-tie error) between the surface and the inertial frames of the vehicle. Correlation of the flash lidar data with the surface elevation data obtained from prior orbiting laser altimeters are particularly reliable in estimating the map-tie error, thereby enabling the TRN algorithm to generate the trajectory correction needed for bringing the vehicle to within 30 meters of the landing target.

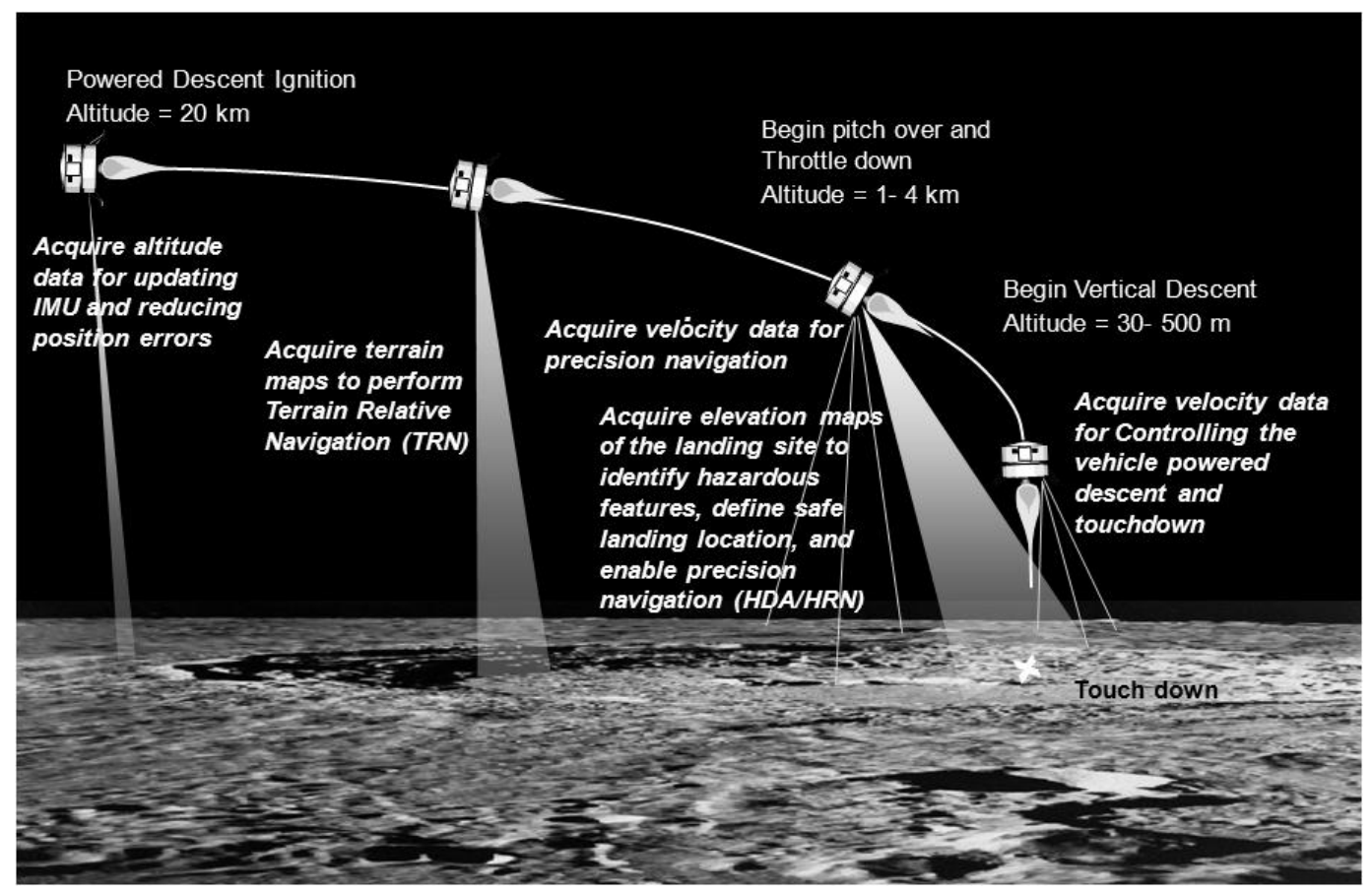

Figure 2: Operational scenario of landing sensors.

After completion of the TRN function at approximately five kilometers above ground level, the flash lidar continues to provide altitude data to help bound the navigation error. At approximately one kilometer in altitude, the laser beam divergence is increased to match the full field of view of the lidar receiver in preparation for the most critical function, HDA)/HRN. The HDA/HRN function requires detection of rocks and surface features greater than $30 \mathrm{~cm}$ in height, detection of slopes greater than $5^{0}$ over the diagonal of the footprint of the landing vehicle, and determination of landmark position to better than one meter relative to the specified landing location (see Figure 3). 
Various concepts for sensor operation and 3-D image acquisition are being evaluated. Each concept must provide the necessary information for achieving the ALHAT HDA/HRN performance goals and simultaneously provide situational awareness information. The primary metric for evaluating an operations concept is robustness, i.e. how efficiently, accurately, and reliably the task is accomplished. In the context of ALHAT, robustness means that the operations concept must be fast, accurate, and be able to provide redundant information for real-time validation of the safe-landing decisions output.

A concept of operation, similar to targeting, that employs some of the capabilities being developed, is termed intelligent safe site selection using precision navigation. Under intelligent safe site selection, HDA/HRN are coupled both in hardware and software since the same process of determining hazard location is also the process for selecting and tracking landmarks for relative navigation meaning that both can be accomplished using the same sequence of 3-D images from the flash lidar. The mode of operation is predicated on the concept that upon recognition of the first available safe landing site, the landing vehicle is directed to it via an appropriate nearby landmark. Continuous operation of the Flash Lidar down to approximately 100 meters above ground level allows for high precision landing within the identified safe landing area, to within approximately one meter. The continuous operation of flash lidar at video frame rates allows the determined safe location to be verified multiple times before the vehicle commits to a touch-down. To ensure that sufficient terrain area is in view throughout vehicle descent, since a fixed field of view (FOV) results in a smaller and smaller footprint as altitude decreases, an adjustable FOV receiver optic is being considered. By increasing the receiver FOV according to its distance to the ground, the spatial coverage and resolution are preserved.
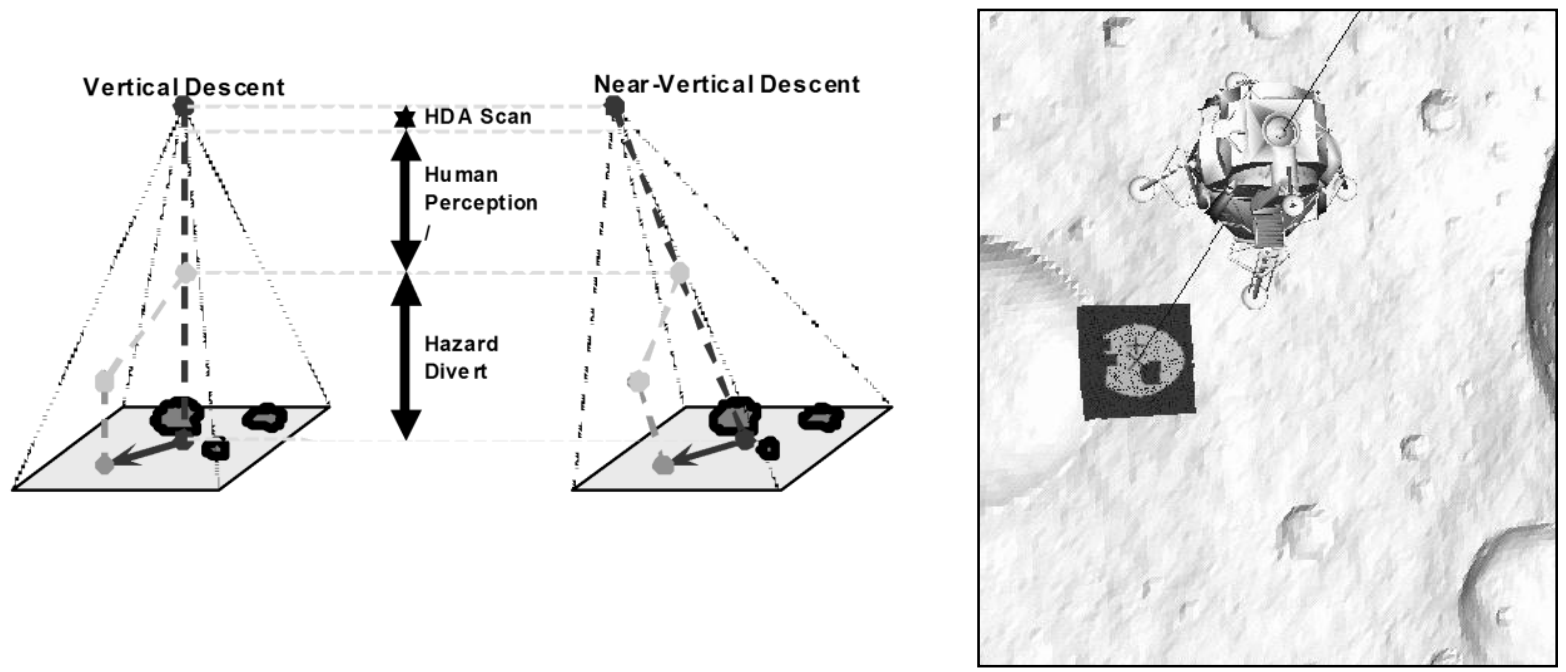

Figure 3: The hazard detection phase is expected to begin when the Lander pitches over to start its approach descent to the surface. The slope of descent can be adjusted to allow for sensor field of view considerations and, if necessary, for human interaction.

The ALHAT project has accomplished three field tests of the proposed systems with the present development targeted for the fourth field test to address the HDA mode of operation. Previous reporting ${ }^{5}$ has detailed the progression toward the ultimate goals as accomplished in each prior development and field test cycle for the flash lidar (field test numbers one and three, with number two being devoted solely to the Doppler lidar). ALHAT's reason for employing two lidars in the present field test number four is to cover two FOV ranges and address two concepts of accomplishing HDA. The first lidar, which has a fixed FOV (fixed-FOV lidar), since its receiver and transmitter optics are set at one degree, is aimed at using a mosaic technique to map the landing site. The small, one-degree FOV is necessary to achieve the spatial precision goal. The second lidar, referred to as the variable-FOV (var-FOV) lidar, is aimed at using a staring technique to map the test site which requires a large enough FOV that mosaic is not needed for scanning around. Since the var-FOV lidar has a large FOV for staring, the spatial precision is degraded which leads to the second objective of the var-FOV lidar which is the demonstration of an image enhancement technique referred to as super-resolution (3-D corollary to digital magnification or zoom in 2-D digital cameras) to improve the spatial precision back to the value achieved in the fixed-FOV lidar via its smaller FOV. The var-FOV lidar is also aimed at developing and testing the 
zoom optics technology which is ultimately intended for zooming out during descent to maintain a large ground footprint for viewing maximum area to select a safe landing site without zooming out enough to compromise the maximum spatial precision number that is tolerable.

\section{LAB CHARACTERIZATION TESTING}

\subsection{Flash Lidar apparatus configuration}

Figure 4 presents the fixed-FOV lidar sensor head which includes the laser head, the fixed transmitter and receiver optics, the 3-D imaging camera all mounted on a liquid-cooled metallic plate. The Nd: YAG Big Sky laser (cooled via liquid through metal tubes for flight) is operated at $20 \mathrm{~Hz}$ to output a $1.064 \mu \mathrm{m}$ pulse $8 \mathrm{~ns}$ Full-Width-Half-Max (FWHM) wide at $30 \mathrm{~mJ}$. Note that the laser (and the full flash lidar system) is capable of operating at $30 \mathrm{~Hz}$; however, $20 \mathrm{~Hz}$ is chosen for compatibility between the fixed-FOV lidar and the ALHAT Navigation Filter in the larger ALHAT GN\&C framework. The Big Sky laser is a class IV, non-eye-safe laser. Two turning mirrors steer the beam through conditioning optics (a 5x beam expander and a top-hat beam shaper) and out the exit aperture of the custom built dust cover. The $1^{\circ}$ receiver optics include a fixed $100 \mathrm{~mm}$ f/7.3 Advanced Scientific Concepts, Inc. (ASC) receiver lens, a plano-concave corrector lens, and a solar filter. The backscatter is sampled by the Tiger Eye 3-D imaging camera serial number 1005 produced by ASC which incorporates a focal plane array (based on an InGaAs APD detector) of 128 x 128 pixels $^{6}$. The two lidars share one data acquisition system (thus only one lidar is operated on a given flight) which encodes the intensity at 8 bits and the range at 16 bits. The system is operated at the lower frame rate of $10 \mathrm{~Hz}$ during most lab characterization in order to minimize cycle time on the instrument during development. The Ground Sample Distance (GSD) of the system at the planned maximum range of $1000 \mathrm{~m}$ is approximately $14 \mathrm{~cm}$ (i.e. the ground footprint of 1 pixel on a normal target) with a maximum ground footprint for the entire array of $17 \mathrm{~m} \mathrm{x} 17 \mathrm{~m}$. The custom-built aerodynamic shroud (used to protect the lidar from the free-stream air and debris during flight) is not shown.

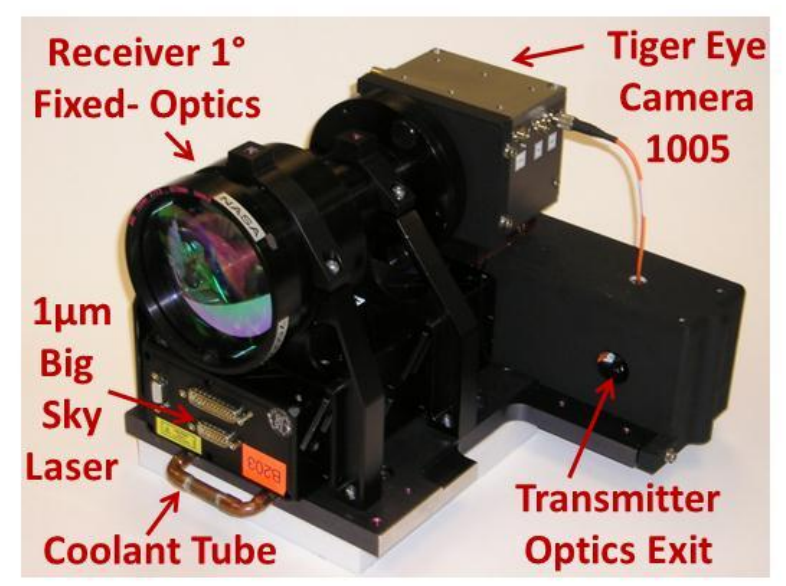

Figure 4: Fixed-FOV lidar sensor head containing the Big Sky laser head, the transmitter optics, the receiver optics, and the 3-D imaging camera along with optics dust cover all mounted on a liquid-cooled metallic plate.

Figure 5 presents the var-FOV lidar sensor head which includes the laser head, the transmitter and receiver optics, the 3D imaging camera all mounted on a liquid-cooled metallic plate. The Nd: YAG Fibertek laser (cooled via liquid through metal tubes for flight) is operated at $30 \mathrm{~Hz}$ to output a $1.064 \mu \mathrm{m}$ pulse $8 \mathrm{~ns}$ (FWHM) wide at $50 \mathrm{~mJ}$. The Fibertek laser is a class IV, non-eye-safe laser. The first and second turning mirrors are used to adjust the beam to pass through the optic center of the three beam expanding lenses. Two of the three beam expanding lenses rest on a linear zoom motor stage so that lens separation is adjusted to achieve the span of desired beam divergence angles which range from $6^{\circ}$ to $16^{\circ}$. The third turning mirror, which is used to align the exit beam with the receiver optics so that the detector is completely illuminated, steers the beam out the exit aperture of the custom built dust cover. The receiver optics include a zoom lens and a solar filter. The $125 \mathrm{~mm} \mathrm{f} / 2.5$ Ball Aerospace zoom lens uses the translation, by a linear motor, of two of its four compound lens element groups to achieve the full range of FOV's extending from $6^{\circ}$ to $24^{\circ}$. Note that the var-FOV lidar is limited to $16^{\circ}$ since the transmitter optics are limited to $16^{\circ}$. The shortfall arose from the mono-static 
transmitter optics delivered by the vendor as part of the zoom lens being non-functional which required LaRC, on short order, to design, develop, characterize, and integrate a set of bi-static transmitter optics into the lidar sensor head which could be automatically zoomed under software control to achieve the desired divergence range. An additional consequence of the vendor not delivering a working set of transmitter optics is that the FOV in flight is limited to one setting in the $6^{\circ}-16^{\circ}$ span as opposed to being able to sweep through a span of FOV's. The setting selected for the flight data is the $6^{\circ}$ setting. Since the f/\# is held constant, less and less of the full aperture is utilized as the FOV increases. The backscatter is sampled by the Tiger Eye 3-D imaging camera serial number 1004 produced by Advanced Scientific Concepts, Inc. which incorporates a focal plane array (based on an InGaAs APD detector) of 128 x 128 pixels $^{6}$. Please refer to the fixed-FOV lidar section for data acquisition details. The system is operated at the lower frame rate of $10 \mathrm{~Hz}$ during most lab characterization in order to minimize cycle time on the instrument during development. The GSD of the system at the planned maximum range of $1000 \mathrm{~m}$ and planned FOV at that range of $6^{\circ}$ is approximately $82 \mathrm{~cm}$ (i.e. the ground footprint of 1 pixel on a normal target) with a maximum ground footprint for the entire array of $105 \mathrm{~m} \mathrm{x} 105 \mathrm{~m}$. The custom-built aerodynamic shroud (used to protect the lidar from the free-stream air and debris during flight) is not shown.

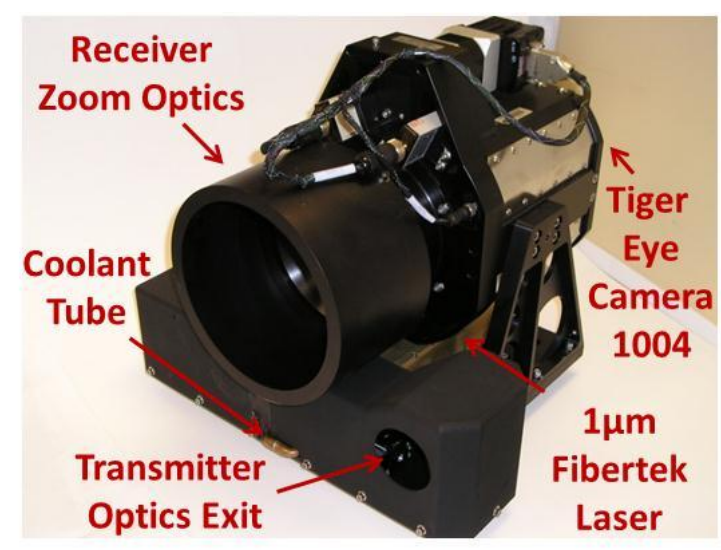

Figure 5: Var-FOV lidar sensor head containing the Fibertek laser head, the transmitter optics (bi-static), the receiver optics (delivered by a vendor with non-functional mono-static transmitter optics which necessitated a short-turn-around bi-static transmitter optics design and development cycle), and the 3-D imaging camera along with optics dust cover all mounted on a liquid-cooled metallic plate.

\subsection{Maximum operational range estimation, Fixed-FOV Lidar}

Standard experimental techniques are used to estimate maximum operational range during lab characterization for comparison to actual flight performance for the fixed-FOV lidar based on the trade of OD for effective range via the lidar equation ${ }^{7}$. The lidar is characterized on the Sensor Test Range (STR) facility at LaRC. Reference 8 provides details on the STR, which is an outdoor test range on which lidars (including non-eye-safe lidars) are fired from an open window overlooking the roof of one building to targets which reside either on the same roof or on that of a neighboring building out to a maximum range of $250 \mathrm{~m}$. The target utilized in the present lab characterization testing is a $1.98 \mathrm{~m}$ flat, square metal board located at a range of 49 meters which is painted white (46\% reflectivity) in a way that its reflectivity at 1.06 $\mu \mathrm{m}$ is diffuse and nearly Lambertian. The fixed-FOV lidar is focused at infinity with depth of field down to $360 \mathrm{~m}$ which results in several pixels of defocus at the target board range which is inconsequential on a flat, uniform board. The camera sensitivity is set at its maximum while still suppressing extraneous backscatter so that no pixels are pretriggered falsely. No aerodynamic shroud was installed since the mechanical development was on-going during lab characterization. The target board of known reflectivity is imaged at zero incidence since reflectivity is a cosine function of incidence with zero incidence yielding maximum reflectivity. The target board is imaged at successive OD values, using a set of one-inch, round ND filters calibrated at the laser wavelength, to effectively attenuate the laser output until no backscatter signal intensity is observed. Based on the known reflectivity value and known incidence angle, data can later be adjusted to more closely match the test site target reflectivity and the imaging angle at a particular point in the trajectory. Transmitter and receiver optics are the flight articles so that reflection and bulk absorption losses are 
indicative of those to be seen in flight. Laser divergence angle and receiver FOV are those to be used in flight, since mismatch between them can be used to artificially amplify or attenuate the reported intensities. The laser divergence settled upon overfills the receiver FOV by approximately 8 milli-radians to provide robustness against any misalignments which may occur as a result of the flight vibration and shock environment. The mosaic technique which is to be evaluated using the fixed-FOV lidar is sensitive to the loss of pixels in the FOV due to such misalignments since the mosaic technique relies on the stitching together of multiple lidar images and hence relies on each image yielding a maximum of illuminated pixels. The transmitter and receiver optics are aligned for flight so that the beam is used most efficiently. The laser is held within its normal thermal operating range using the active, liquid cooling system for a pulse energy output of $31.3 \mathrm{~mJ}$ at the laser output coupler and for a pulse energy output of $23.6 \mathrm{~mJ}$ emanating from the last transmitter optic.

The lab characterization results indicate the maximum operational range to be approximately 1,070 meters. The criterion applied in the present case is that greater than $90 \%$ of the pixels should trigger at the OD test point designated as the maximum operating range condition, with maximum range being that condition at which only approximately $90 \%$ of the pixels trigger. Figure 6, which is a plot of the percentage of triggered pixels as a function of OD for images taken of the STR target at an actual range of $49 \mathrm{~m}$, indicates that an OD of 2.68 corresponds to the $90 \%$ condition. Recall that the detector array contains 16,384 pixels in an array of 128 by 128 .

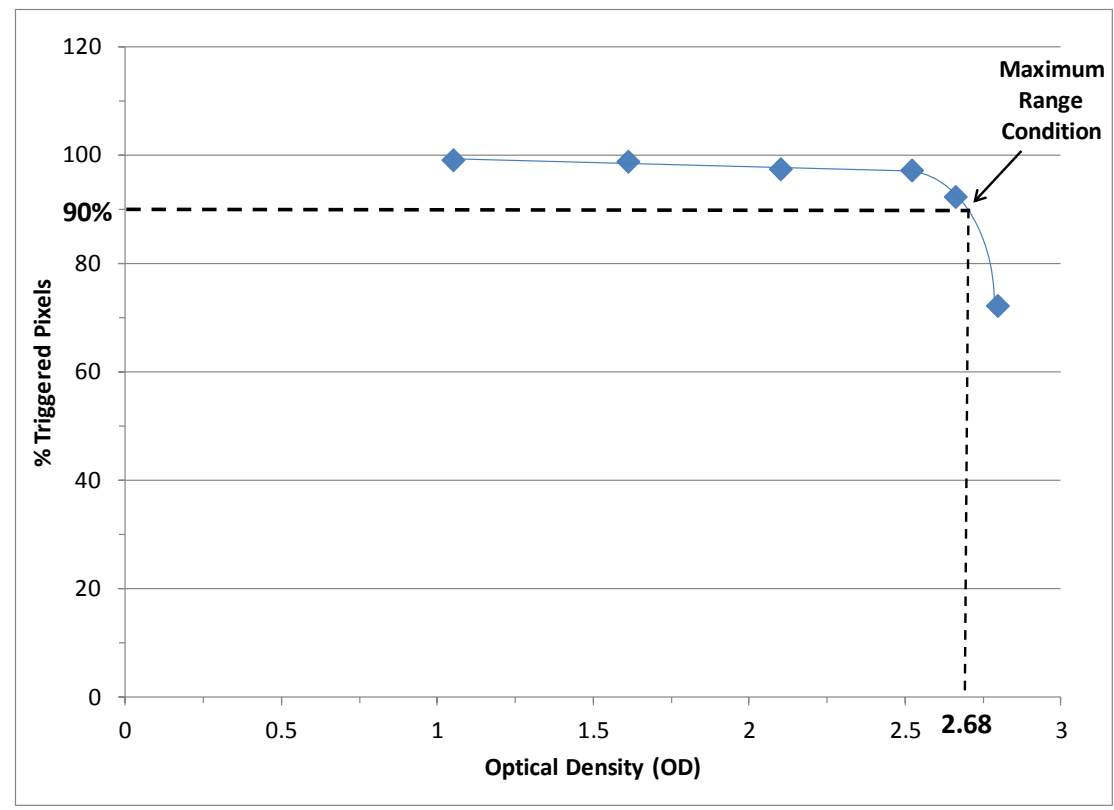

Figure 6: Maximum operating range estimated to be $1070 \mathrm{~m}$ for fixed-FOV lidar identified at $90 \%$ triggered pixels level for OD = 2.68 for images taken of STR target (46\% reflectivity) at actual range of $49 \mathrm{~m}$.

Figure 7 presents the intensity and range image contour plots of the target at $49 \mathrm{~m}$ for the closest OD case that is at or above the maximum-range OD value of 2.68 which turns out to be the OD case of 2.80. The contour plots of Figure 7 show pixel number on the $\mathrm{x}$ and $\mathrm{y}$ axes (128 pixels on each axis) with one plot of Figure 7 depicting intensity in counts as the contour variable (with noise floor at 1000 counts and the upper limit at 4096 counts) and with the other plot depicting range in meters as its contour variable. Figure 7 shows that most pixels are triggered and yield intensity data in the vicinity of 1500 counts which is above the noise floor of 1000 counts to provide enough signal-noise ratio from which to develop a proper range solution. Figure 7 also shows that, indeed, precise range values are generated around 54.2 meters. The lidar-indicated range to the target is approximately $54 \mathrm{~m}$ instead of the actual $49 \mathrm{~m}$ and is due to range accuracy biasing that is not critical for the hazard detection objective of the present development and field test cycle. 

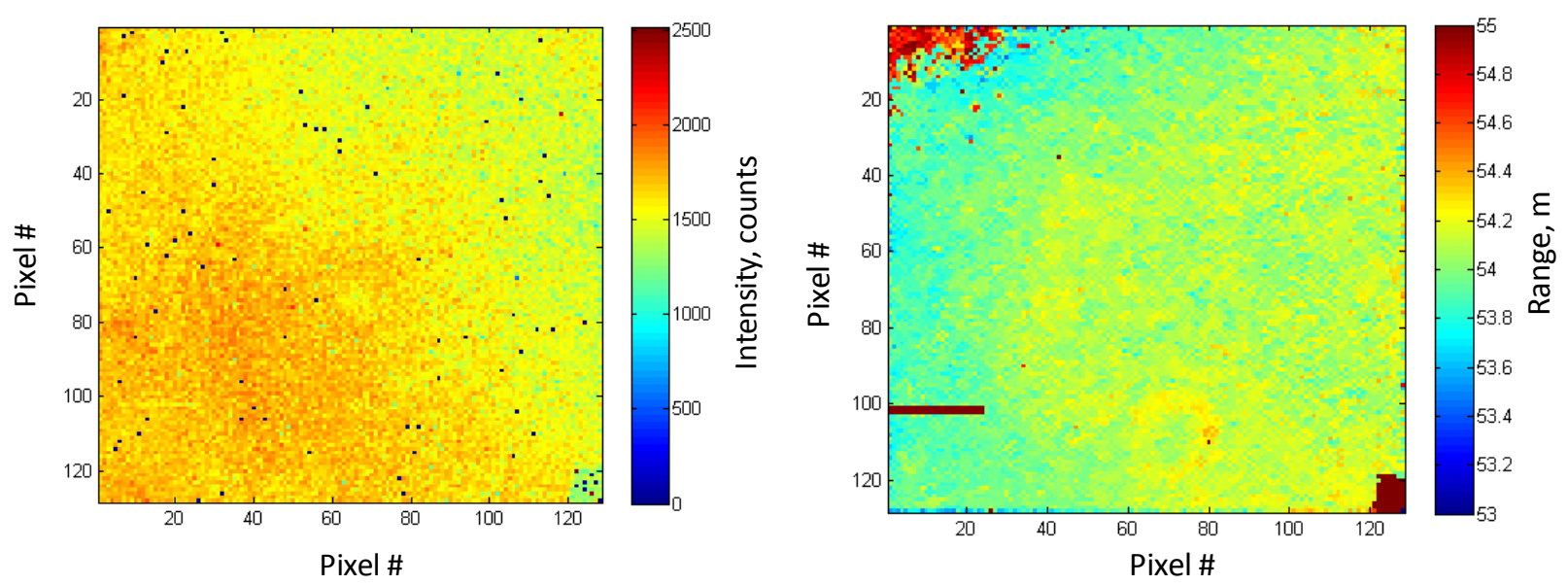

Figure 7: Intensity and range contour plots of $49 \mathrm{~m}$ target board ( $46 \%$ reflectivity) for fixed-FOV lidar at OD $=2.80$ which is the nearest contour plot which is at or above the maximum operational range case.

\subsection{Maximum operational range estimation, Var-FOV Lidar}

Standard experimental techniques are used to estimate maximum operational range during lab characterization for comparison to actual flight performance for the var-FOV lidar based on the trade of OD for effective range via the lidar equation ${ }^{7}$. The var-FOV lidar is also characterized on the STR facility at LaRC, please refer to the STR discussion in the fixed-FOV section for details on the test range and the target board used. The var-FOV lidar is focused at infinity with depth of field down to $37 \mathrm{~m}$. The camera sensitivity is set at its maximum while still suppressing extraneous backscatter so that no pixels are pre-triggered falsely. No aerodynamic shroud was installed since the mechanical development was on-going during lab characterization. The technique of trading OD for effective range is the same as that described in the fixed-FOV lidar section with the only difference being that a four inch square ND filter set is used, which is also calibrated at $1.06 \mu \mathrm{m}$. Transmitter and receiver optics are the flight articles so that reflection and bulk absorption losses are indicative of those to be seen in flight. The transmitter and receiver optics are set for FOV's of $6^{\circ}, 7.5^{\circ}, 10.6^{\circ}$, and $13.5^{\circ}$. Laser divergence angle and receiver FOV are those to be used in flight, since mismatch between them can be used to artificially amplify or attenuate the reported intensities. The laser divergence settled upon overfills the receiver FOV by approximately 8 milli-radians to provide robustness against any misalignments which may occur as a result of the flight vibration and shock environment. The transmitter and receiver optics are aligned for flight so that the beam is used most efficiently. The laser is held within its normal thermal operating range using the active, liquid cooling system for a pulse energy output of $50 \mathrm{~mJ}$ at the laser output coupler.

The lab characterization results indicate the maximum operational range to equal or exceed 1,600 meters. A usual rule applied in flash lidar testing is that greater than $90 \%$ of the pixels should trigger at the OD test point, however, due to the wide FOV's of the var-FOV lidar, only a subset of the FOV can be tested on the STR. The maximum range for the varFOV lidar is considerable larger than the goals for FT $4(1,000 \mathrm{~m})$ and larger than the maximum value which can be represented by the LaRC flight software since it is built around the same FT 4 goals. Thus, the conservative criteria for determining maximum range selected for the present case are that all pixels representing the range target should be illuminated for all FOV settings at a signal to noise value of 1.3 or greater. The stipulation that the criteria be satisfied for all FOV's arises from the constant f/\# at which the receiver zoom optics operates. Since the f/\# is held constant through all FOV's (i.e. focal lengths), then the aperture is reduced at successively larger FOV's (smaller focal lengths) and, since lens throughput is dominated by aperture, the intensity for the same OD's at larger and larger FOV's will be reduced. Figure 8 presents intensity and range image contour plots of the target at $49 \mathrm{~m}$ for the $6^{\circ} \mathrm{FOV}$ case at the maximum range $\mathrm{OD}$ value of 3.05. The contour plots of Figure 8 show pixel number on the $\mathrm{x}$ and $\mathrm{y}$ axes (128 pixels on each axis) with on plot depicting intensity in counts as the contour variable (with noise floor at 1000 counts and the upper limit at 4096 counts) and with the other plot depicting range in meters as its contour variable. Figure 8 shows that most (> 90\% certainly) are triggered and yield intensity data in the range of 2100 counts which is well above the noise floor of 1000 counts to provide enough signal-noise ratio from which to develop a proper range solution. Figure 8 shows 
that, indeed, precise range values are generated around 48.7 meters. Note that since the Fibertek is the planned ALHAT space laser, the maximum range achieved with it is the defining case for the maximum range objective from an ALHAT standpoint.
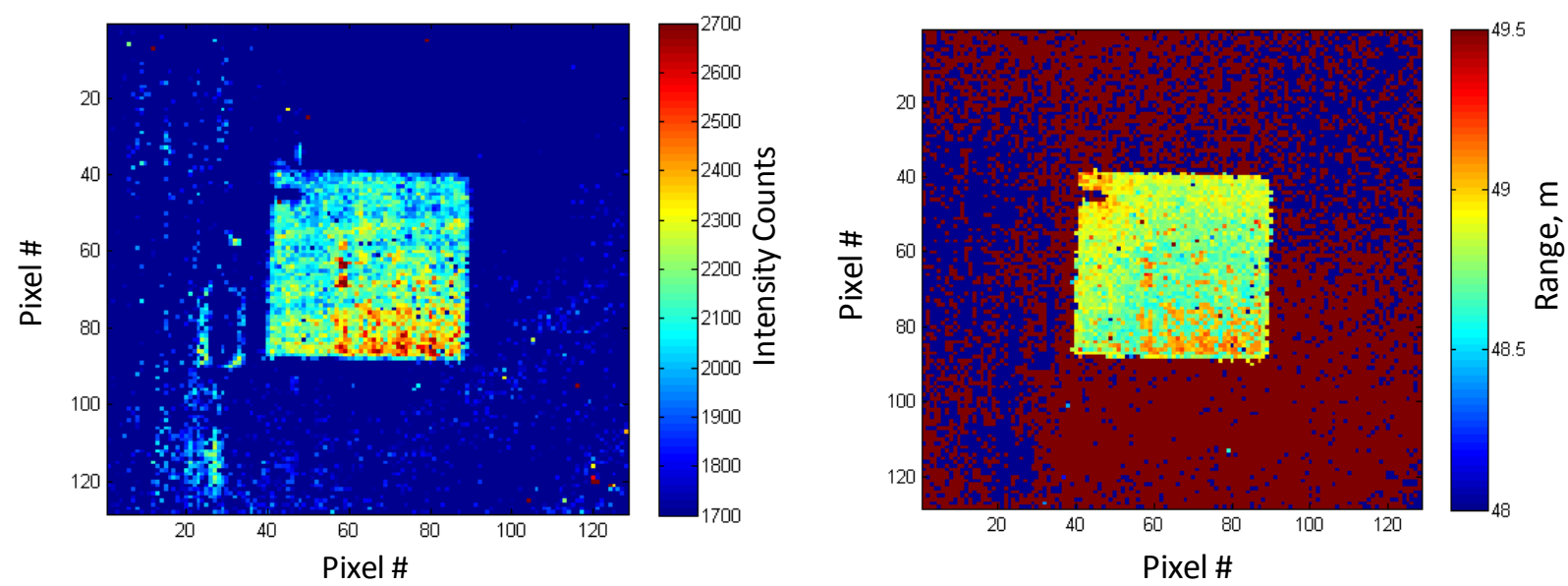

Figure 8: Intensity and range contour plots of $49 \mathrm{~m}$ target board (46\% reflectivity) at $6^{\circ} \mathrm{FOV}$ for var-FOV lidar at OD $=3.05$.

\section{HELICOPTER INTEGRATION AND FLIGHT TEST PLAN}

The NASA-LaRC lidars along with supporting electronics are integrated and tested along with the rest of the ALHAT GN\&C system components from NASA-JPL, NASA-JSC, and Draper Labs into an instrumentation pod (see Figure 9) on the mesa test range at NASA JPL. The flash lidar sensor heads, along with the Doppler Lidar and Laser Altimeter, are installed in a vertically translating rack structure (see Figure 9) within the pod so that they can be either deployed in flight and pointed to the desired target area with the two-axis gimbal or retracted within the pod and the pod belly door closed during hazardous portions of the flight (takeoff and landing) or any time that laser safety must be ensured. The ALHAT systems are self-contained in the pod by design so that all checkout operations are performed separately from the helicopter so that all debugging can occur prior to integration with the helicopter. Successful ranging on both normal and non-normal incidence flat target boards on the JPL mesa test range is accomplished for the fixed-FOV lidar (with aerodynamic shroud not installed) at $975 \mathrm{~m}$. 

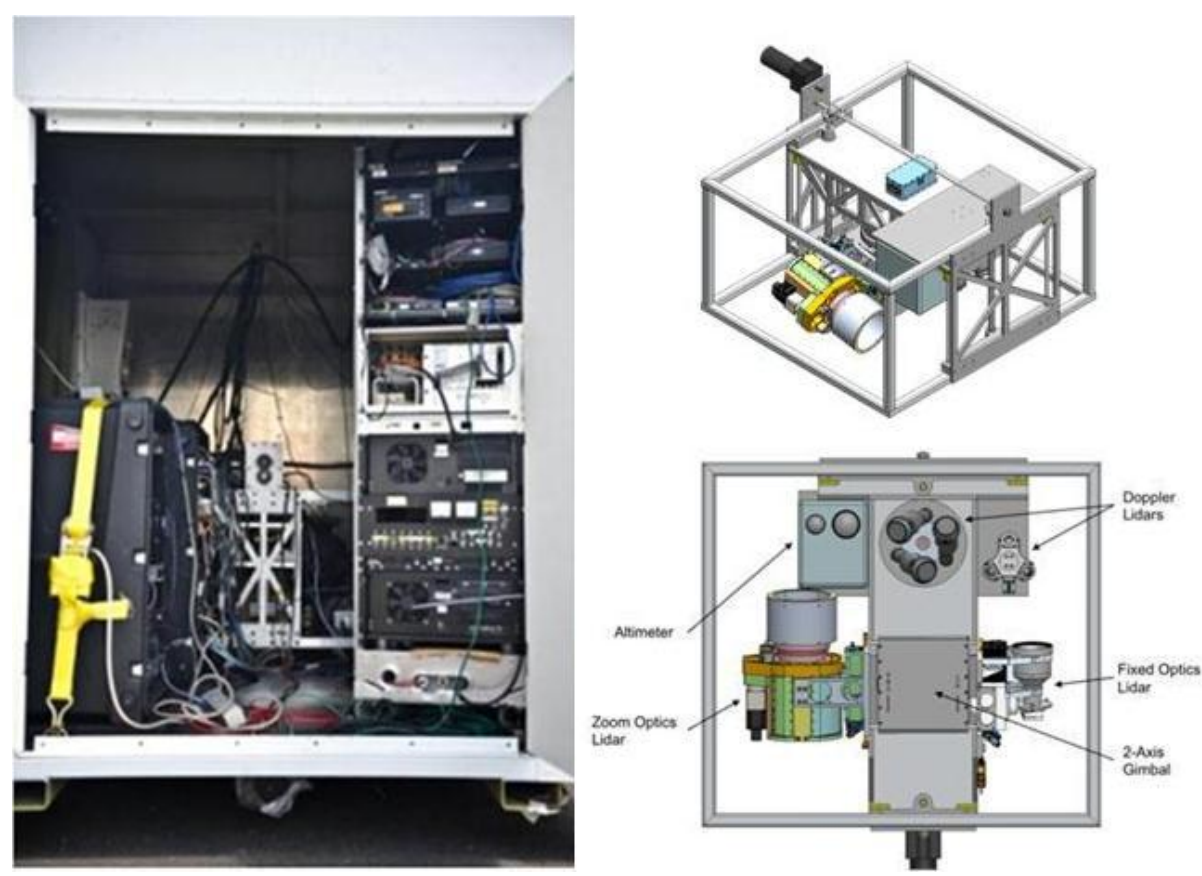

Figure 9: Instrumentation pod interior (left) showing 19-inch rack mount electronics and rack structure inside the pod (right) which retracts the lidars up into the pod when not in use so the protective belly door can be closed.

After instrumentation pod integration and testing activities are completed at NASA-JPL, the pod is moved to the high desert to be integrated to the Erickson Aircrane helicopter at NASA-Dryden. The pod attaches to the helicopter (Figure 10) via a set of hooks just as other equipment attaches to the helicopter during its normal fire-fighting operations. Cabling for operator interface equipment is run from the pod to the aft-facing, aft cockpit of the helicopter where an ALHAT systems operator resides in place of the usual crane operator. Figure 10 shows both lidars as deployed under the pod with aerodynamic shrouds installed and laser exit apertures labeled.

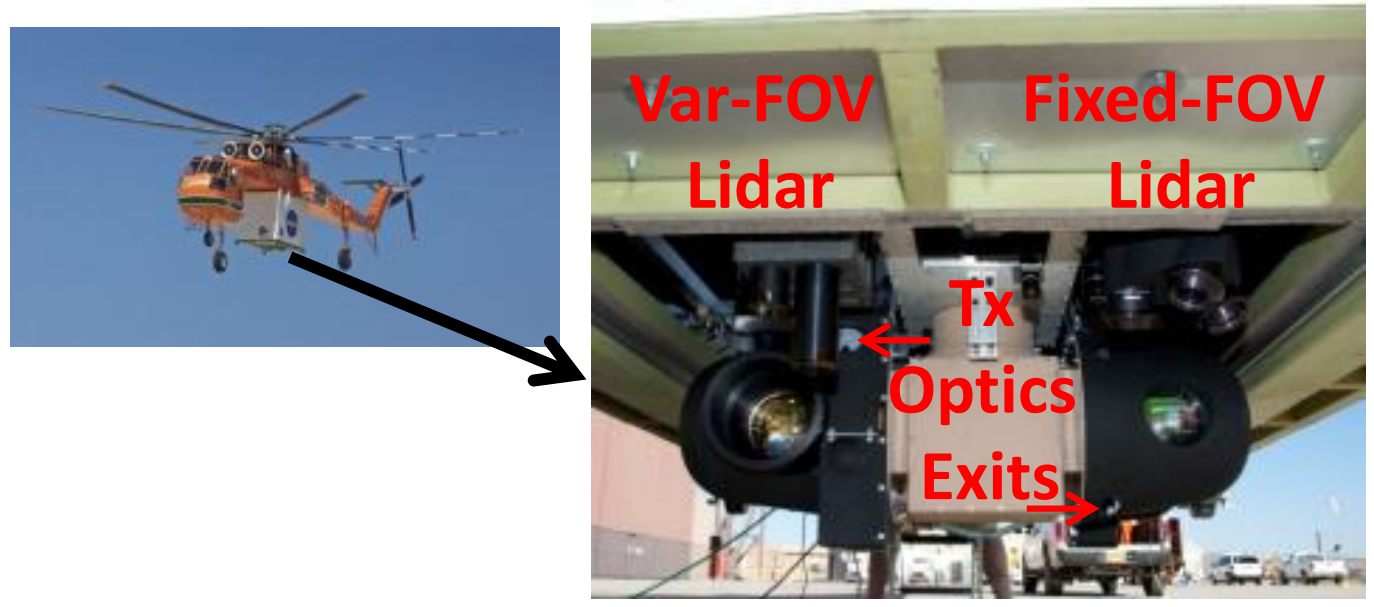

Figure 10: Instrumentation pod installed on Erickson Aircrane helicopter (left) lifting off from NASA-Dryden and en-route to Edwards AFB test range carrying the ALHAT instrumentation pod with pod belly doors closed and the lidars retracted. The right image shows the pod doors open and lidars deployed with protective aerodynamic shrouds on each and laser exit apertures noted. 
Field test \#4 helicopter flight test operations consist of multiple approaches to a hazard field of human-made geometric targets. The Erickson Aircrane helicopter is chosen for its large payload capacity (in service, it acts as a powerful helicopter-based crane for heavy-lift operations) and resulting excess power which enables it to achieve trajectories utilized in the ALHAT planetary landing simulations. Flights originate from NASA-Dryden in the upper portion of Figure 11 and proceed to the hazard field situated several miles away on the southwest corner of the Edwards AFB Rogers Dry Lake. Figure 11 shows a portion of the hazard field which consists of a host of square and hemispherical targets of varying size and spacing as well as sloped hazard areas and safe-sites with no hazards. Additional characterization runs target the surrounding flat lakebed surface.

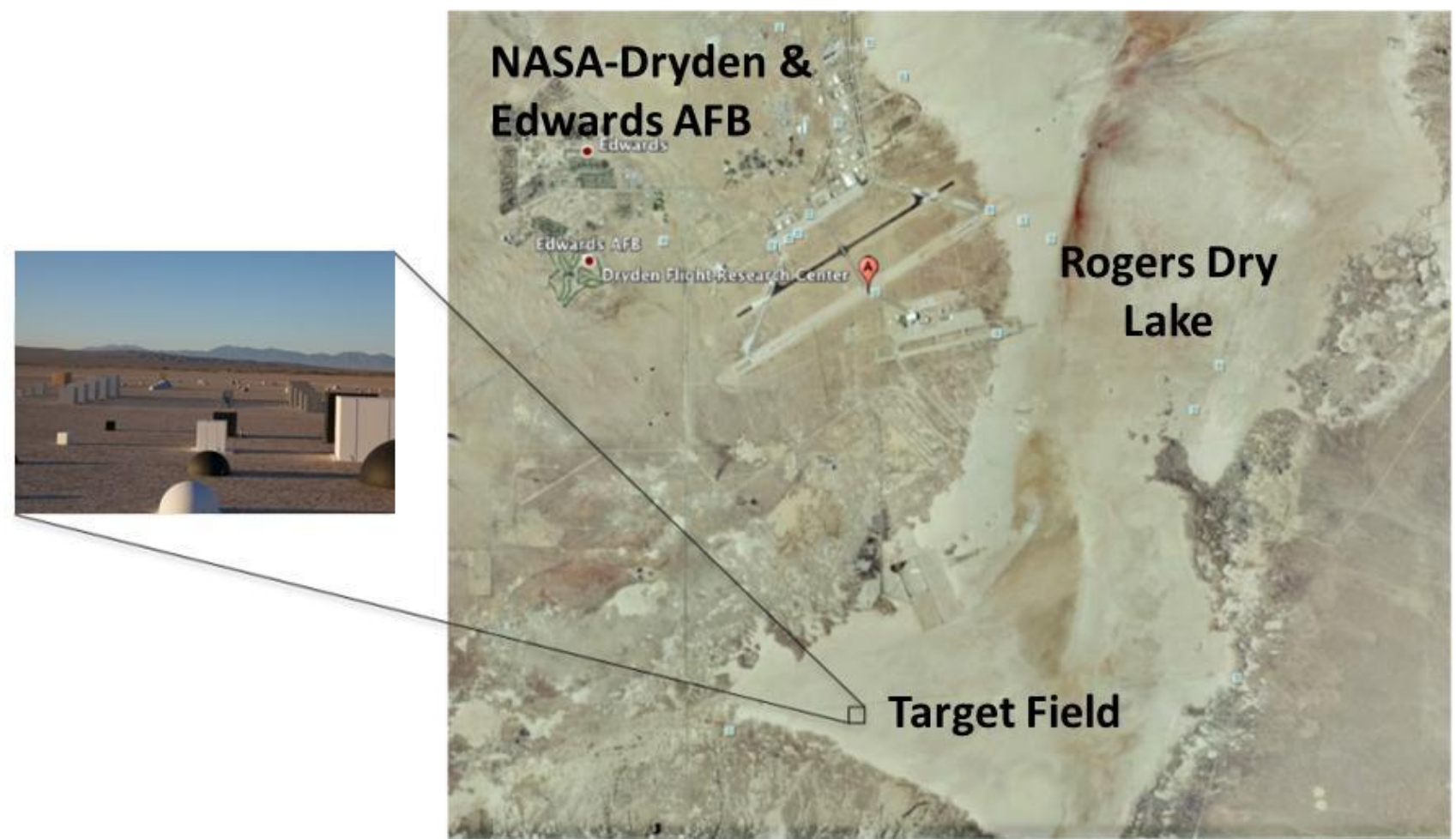

Figure 11: Edwards AFB Rogers Dry Lake with hazard field situated on the southwest corner. An aerial close-up of the target hazard field is shown (containing a host of square, hemisphere, and slope hazards as well as safe sites).

\section{HELICOPTER FLIGHT TEST RESULTS}

A number of cutting-edge components from industry and NASA are integrated, many for the first time, into two flash lidar sensors which take the next step toward the final ALHAT system for space missions via atmospheric flight testing. The field test consists of eight flights spread over a week in the summer of 2010. The first three flights are referred to as shakeout flights and are focused mainly on initial checkout of systems in flight, although usable data is gleaned from the flights. Table 2 lists the flights along with the lidar under test (since the support electronics can only drive one lidar system at a time) along with pertinent notes. In shakeout flight two, the systems performed nominally and the data is usable for characterization. Fiber optic t0 damage on the var-FOV lidar minimized the useful data available from the third shakeout flight and kept the var-FOV lidar in an inoperative state until the final flight of the campaign. Flight numbers one through three, which employed the fixed-FOV lidar, generated nominal data usable for characterization. Flight number four is dedicated to camera and gimbal objectives and the lidars are not operated. With the repair to the var-FOV lidar's t0 accomplished just prior to flight, no time was available for optimization and thus the final flight of the campaign served as a basic functional checkout of the in-flight performance of the var-FOV lidar. LaRC custom data acquisition and control flight software bugs resulted in intermittent performance and data storage over all flights. The JPL two-axis gimbal (critical to hazard detection since it ensures that the lidar is pointed at the hazard field) experienced similar intermittent performance due to unresolved system bugs. As a result of the anomalies in the full, integrated 
ALHAT system, sufficient data is not available to make conclusions on hazard detection and avoidance, although sufficient data is gleaned in order to characterize the lidars.

Table 2: Flight log from ALHAT field test \#4.

\begin{tabular}{|c|c|c|}
\hline Flight Number & $\begin{array}{c}\text { Flash Lidar } \\
\text { Utilized }\end{array}$ & Notes \\
\hline Shakeout Flight 1 & Fixed-FOV & No data collected, 7/25/2010 \\
\hline Shakeout Flight 2 & Fixed-FOV & $7 / 26 / 2010$ \\
\hline Shakeout Flight 3 & Var-FOV & Fiber optic t0 damage, $7 / 26 / 2010$ \\
\hline Flight 1 & Fixed-FOV & $7 / 27 / 2010$ \\
\hline Flight 2 & Fixed-FOV & $7 / 27 / 2010$ \\
\hline Flight 3 & Fixed-FOV & $7 / 28 / 2010$ \\
\hline Flight 4 & None & Lidars not operated, $7 / 28 / 2010$ \\
\hline Flight 5 & Var-FOV & $7 / 29 / 2010$ \\
\hline
\end{tabular}

Lidar image processing consists of several real-time and post-processing steps. Real-time image construction consists of peak detection in order to establish a time-of-flight range solution and subsequent application of range / intensity calibration to improve the range solution. The post-processing steps (which occur after the flight) include removal of bad pixels using the bad pixel map as a guide. Each Lidar range image is converted to a DEM. One example of one of the many steps involved in conversion of a lidar range image to a DEM is the removal of vehicle attitude perspective which introduces a sloping effect to the terrain prior to correction.

Interpretation of maximum range data builds upon the interpretation methods used in the lab characterization section. Maximum operational range is defined as the range where $90 \%$ of the pixels trigger. The LaRC custom data acquisition system represents range with a 16 bit word using a two centimeter step size which sets 1300 meters as the largest range number that can be represented. The LaRC custom data acquisition system represents intensity with an 8 bit word with a step size of 16 intensity counts giving the full 0 to 4096 intensity counts span which is available from the camera.

\subsection{Maximum operational range flight results, Fixed-FOV lidar}

The flight results define a maximum range for the fixed-FOV lidar of $600 \mathrm{~m}$. Figure 12 shows the percentage of triggered pixels for each complete frame of lidar data as a function of the frame number. The frames are acquired at 20 $\mathrm{Hz}$ (reduced from $30 \mathrm{~Hz}$, of which the flash lidar is capable, for compatibility with the ALHAT navigation filter for the fixed-FOV lidar only) indicating that the descent maneuver lasts approximately five minutes. Since the maximum range is defined as the range at which $90 \%$ of the pixels trigger, the maximum range occurs approximately at frame number 4300. Thus for frame numbers less than 4300 , less than $90 \%$ of the pixels trigger which means that the range image is only a partial image. 

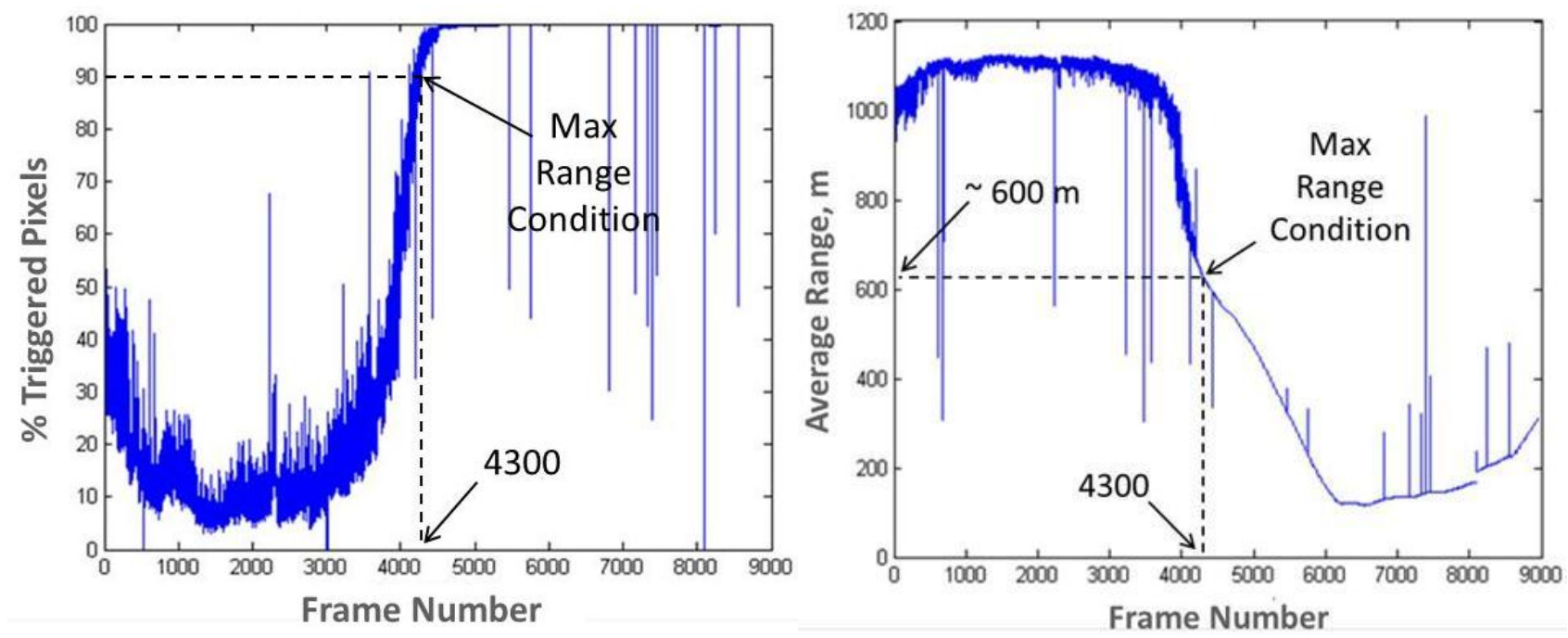

Figure 12: Shakeout flight \#2 (fixed-FOV lidar) descent maneuver percentage of triggered pixels and average range as a function of frame number at a rate of 20 frames / sec. The maximum range (90\% pixels triggered) of approximately $600 \mathrm{~m}$ is shown to occur in the vicinity of frame number 4300 .

Figure 13 presents an intensity contour plot along with its associated range contour plot for a range value above the maximum range condition as defined in Figure 12 in order to provide a sample of the behavior of the lidar above maximum range. The intensity contour in Figure 13 shows that only the lower left corner of pixels trigger which is indicated by an intensity value at slightly above the noise floor of 1000 counts. The corresponding range contour plot confirms that only a cluster of pixels in the lower left trigger and they report a range value around $800 \mathrm{~m}$ while the remainder of the un-triggered pixels just automatically report full scale on the range word (the camera outputs $2130 \mathrm{~m}$ which is represented by the LaRC custom data acquisition system as $1300 \mathrm{~m}$ ) which is outside the bounds of the displayed range contour plot.
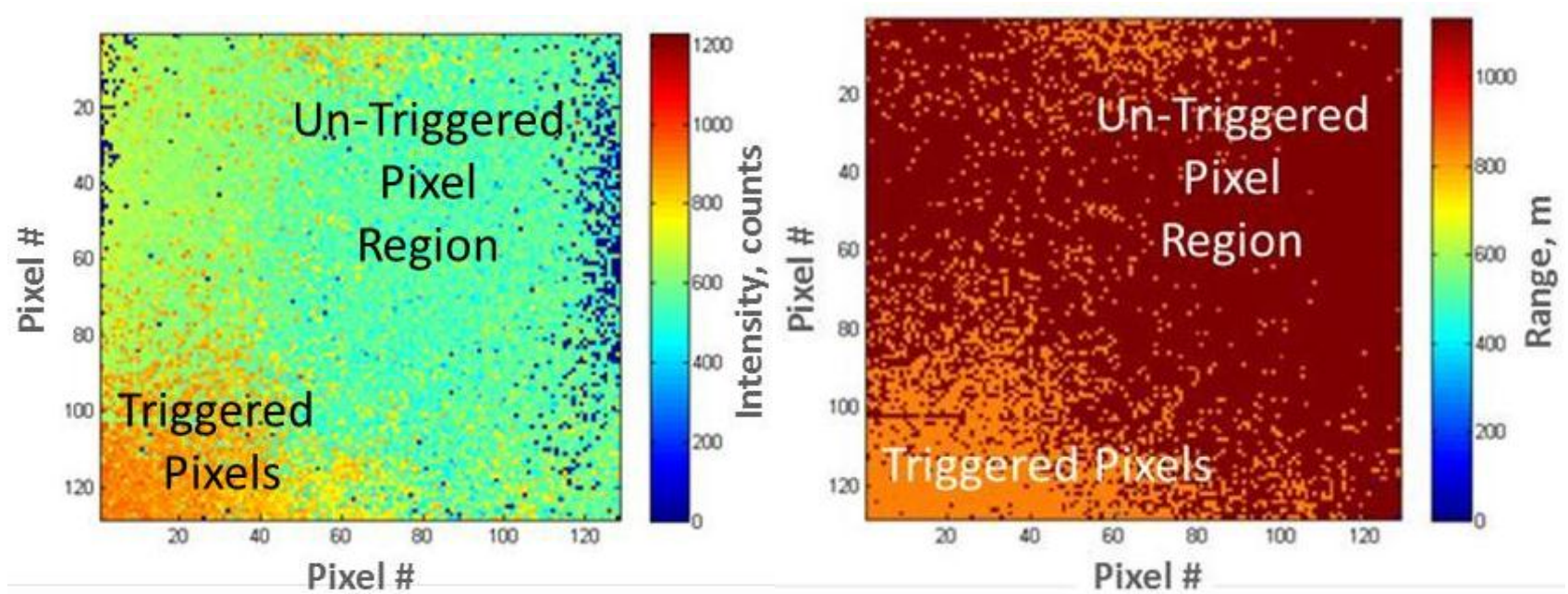

Figure 13: Shakeout flight \#2 (fixed-FOV lidar) intensity and range contour frames at a range above the maximum range of $600 \mathrm{~m}$. Image depicts some triggered (lower left) and some un-triggered pixels with un-triggered pixels resulting from the range being slightly above the maximum range condition. Most intensity values are outside the linear range.

Figure 14 presents an intensity contour plot along with its associated range contour plot for a range value below the maximum range condition as defined in Figure 12 in order to provide a sample of the behavior of the lidar below maximum range. The intensity contour in Figure 14 shows that all pixels trigger which is indicated by an intensity value 
at or above the noise floor of 1000 counts. Most pixels indicate an intensity value somewhere between 1100 and 1600 counts. The corresponding range contour plot in Figure 14 confirms that all pixels trigger and they report a range value around $322 \mathrm{~m}$. A range gradient appears in the range contour plot. The gradient is just the apparent ground slope introduced by gimbal / vehicle attitude being non-normal to the lakebed being imaged.

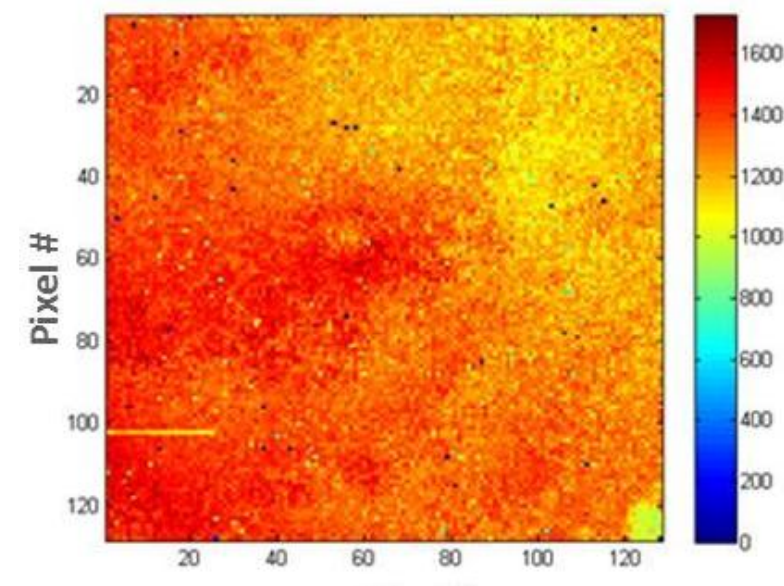

Pixel \#

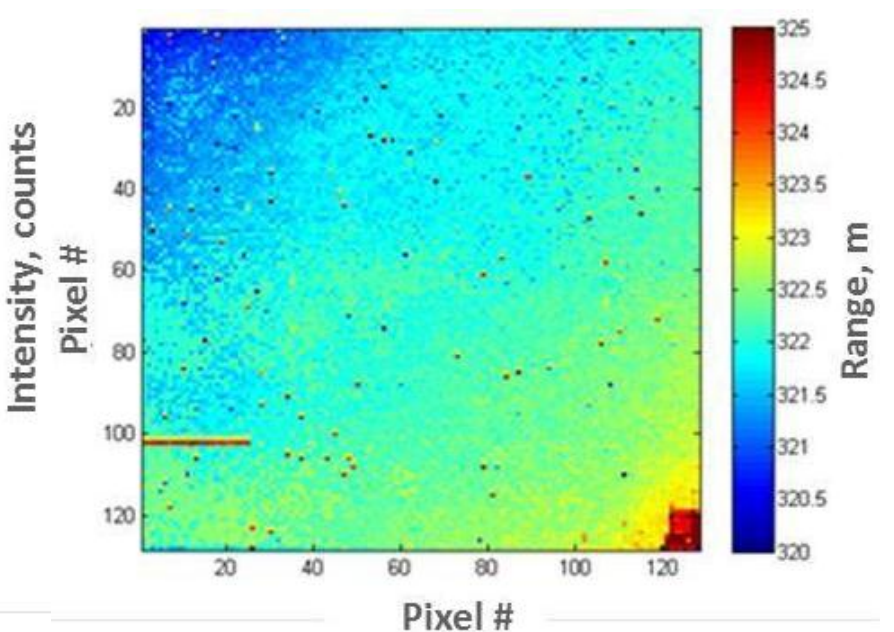

Pixel \#

Figure 14: Shakeout flight \#2 (fixed-FOV lidar) intensity and range contour frames at a range of approximately $322 \mathrm{~m}$ which is below the maximum. Noise floor is at 1000 counts. The image depicts triggering of all pixels. All intensities are within the linear range. The range gradient in the frame is due to vehicle / gimbal attitude, i.e. the lakebed has an apparent slope due to a non-zero-degree incidence angle between the ground and the lidar.

Since the lab characterization testing predicts the maximum operating range to be $1070 \mathrm{~m}$, the $600 \mathrm{~m}$ range measured in flight represents a significant shortfall. The shortfall is due to unexpected laser scatter from a last minute replacement of the aerodynamic shroud that is used to protect the lidar sensor head during flight. The previous shroud had too much damage to be flight-worthy due to the shipping process and all of the removals and reinstallations which occurred during the integration testing. The exit hole for the beam in the new shroud was not aligned properly with the optics which resulted in some laser energy being lost due to scatter. Maximum range was further reduced since the scatter caused some pixel pre-triggering which necessitated a manual reduction in the lidar's sensitivity. If not for the aerodynamic shroud hole being slightly offset, it is likely that the fixed-FOV lidar would have achieved the predicted lab performance (further supported by some operational testing accomplished during integration at JPL in which the lidar imaged an actual target at $975 \mathrm{~m}$ with extra signal strength to spare).

\subsection{Target detection, Fixed-FOV lidar}

Several targets of opportunity are detected. Due to the combination of lidar data acquisition glitches and gimbal anomalies, the integrated system did not operate simultaneously for long enough to accomplish purposeful hazard detection at the ranges which would have stressed the sensor and algorithms. Figure 15 shows a $38 \mathrm{~cm}$ hemispherical hazard imaged on the second shakeout flight from a range of $325 \mathrm{~m}$. Figure 15 shows the intensity and range images of the hazard. The intensity image shows the hazard and surrounding ground to be in the linear range and the range image shows an apparent slope which is due to vehicle attitude with respect to the flat, un-sloping ground. The DEM of Figure 15 removes the apparent slope. Note that the $38 \mathrm{~cm}$ hemisphere is just a smaller hemisphere in a line of hemispheres decreasing in size which is made clearer by the picture in Figure 16 showing the subject set of hazards positioned on the Rogers Dry Lake. Figure 17 is a range contour plot showing a stack of boxes from flight number three at a range of approximately $180 \mathrm{~m}$. 

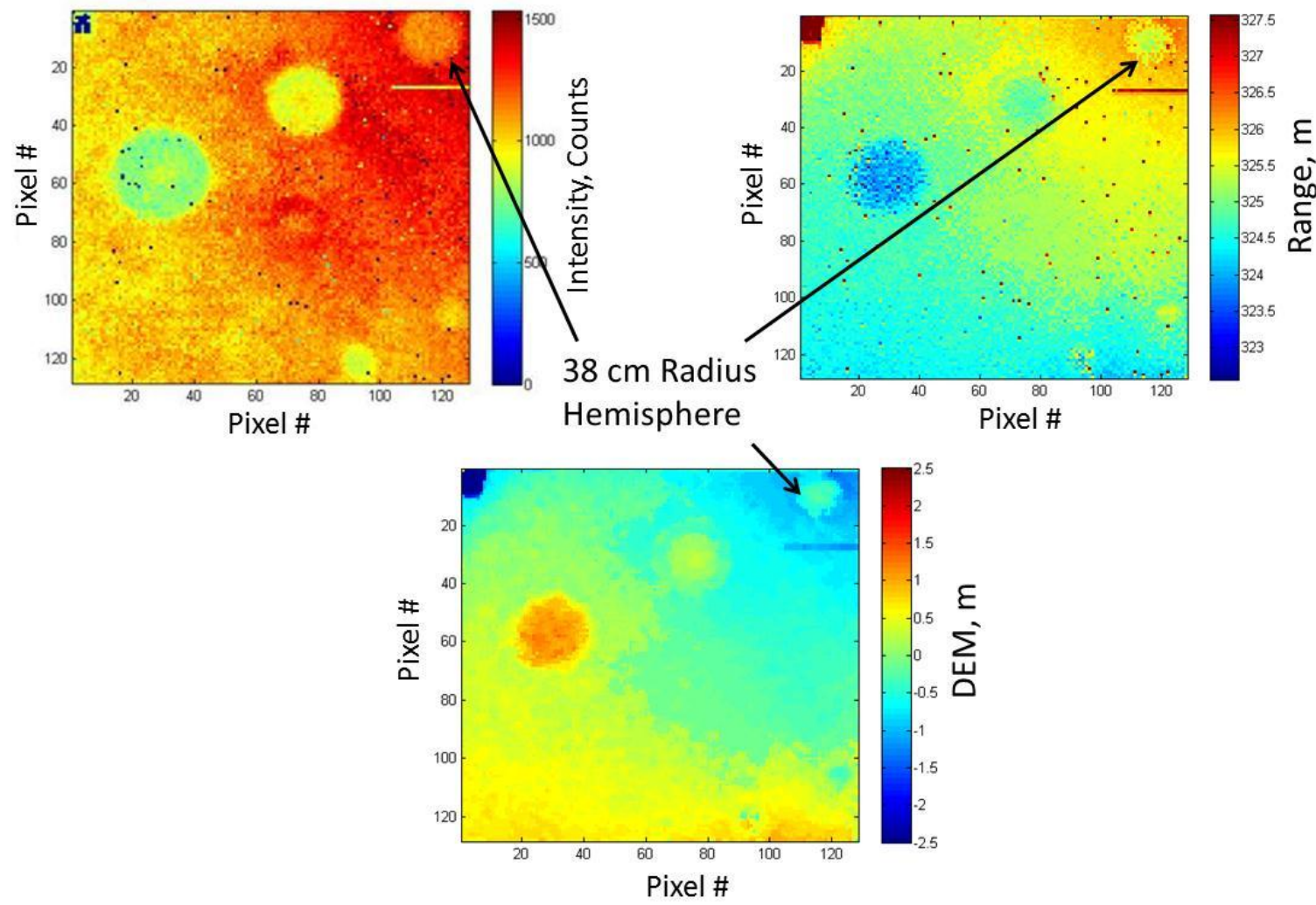

Figure 15: Shakeout flight \#2 (fixed-FOV lidar) $38 \mathrm{~cm}$ hemisphere target detection at a range of $325 \mathrm{~m}$. The intensity image, the range image, and the DEM are shown. All intensities are within the linear range. Pixel size is $4.5 \mathrm{~cm}$.

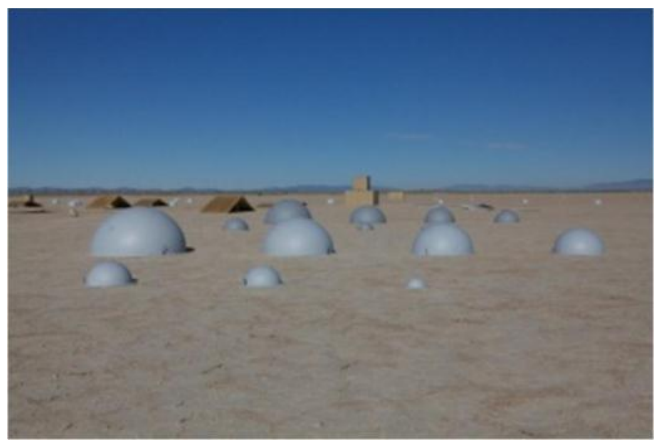

Figure 16: Hemispherical targets on Rogers Dry Lakebed, Edwards AFB, CA as detected in previous figure. 


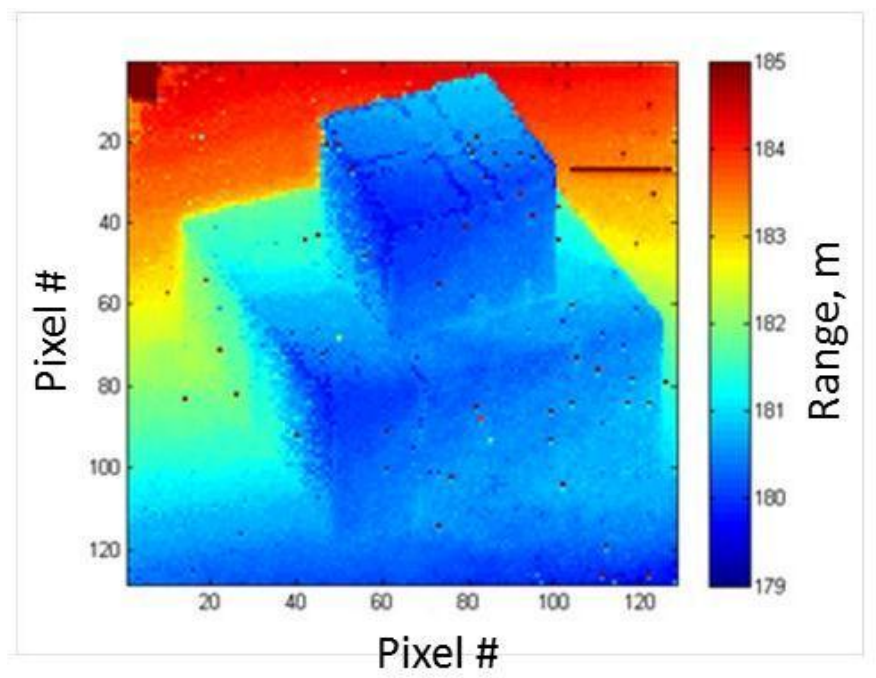

Figure 17: Flight \#3 (fixed-FOV lidar) target detection (stacked boxes) range image. Pixel size is $2.5 \mathrm{~cm}$.

\subsection{Maximum operational range flight results, Var-FOV lidar}

The var-FOV lidar exceeded the FT 4 maximum operational range goal of $1 \mathrm{~km}$ despite a t0 anomaly which arose during its first flight of the campaign and which precluded successful flight operations until its repair was accomplished just prior to the final flight (\#5) of the campaign. No time was available for pre-flight optimization. Since no time was available for long-range, on-ground alignment, a portion of the var-FOV lidar's pixels were not illuminated and thus remained un-triggered. An additional consequence of no pre-flight testing was a data acquisition system anomaly which caused a loss of data for approximately $25 \%$ of the pixels. Figure 18 presents an intensity contour plot along with its associated range contour plot at the six degree FOV setting for a range value of approximately $1,090 \mathrm{~m}$, which is slightly below the maximum range condition, in order to provide a glimpse into the behavior of the var-FOV lidar below maximum range. The intensity contour in Figure 18 shows the strip of lost data on the right (lost due to the data acquisition system anomaly) and the un-triggered pixels around the perimeter. The un-triggered pixels fall at and below the noise floor of 1000 counts. The intensity contour in Figure 18 shows that all triggered pixels fall in the linear region. The corresponding range contour plot in Figure 18 confirms the strip of lost data and the un-triggered pixels. In the case of the range plot, un-triggered pixels manifest themselves as a full scale range value which exceeds the contour maximum. A range gradient appears in the interior, triggered-pixel portion of the range contour plot. The gradient is just the apparent ground slope introduced by gimbal / vehicle attitude being non-normal to the lakebed. 

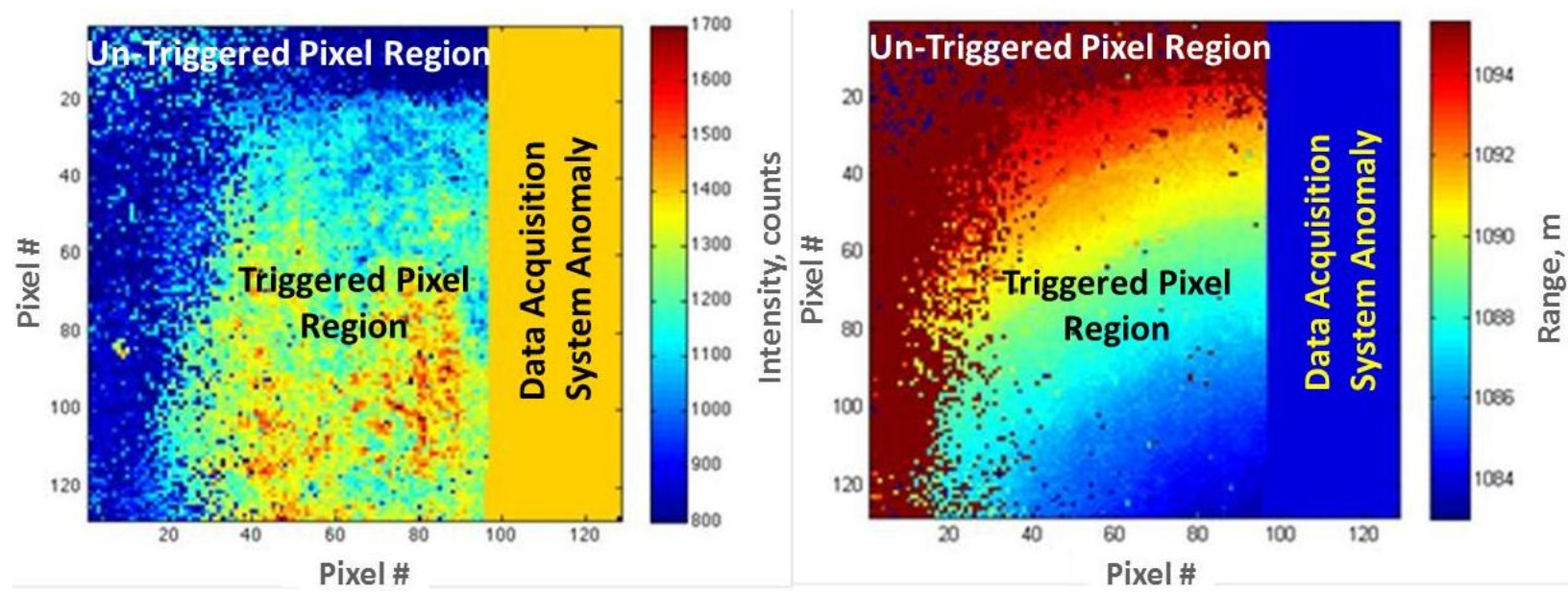

Figure 18: Flight \#5 (var-FOV lidar) intensity and range contour frames for the six degree FOV setting at a range of approximately 1 $\mathrm{km}$ showing the strip of lost data on the right side of each image and the un-triggered pixels (near the 1000 count noise floor) at the left and top of each image which results from transmitter / receiver optics misalignment. The images' interior portion show good intensity data (in the linear range) and range data whose expected sloping character results from the non-zero-degree incidence angle between the lidar and the ground.

The flight five results define a maximum range for the var-FOV lidar of approximately $1,200 \mathrm{~m}$. The strip of lost data and the un-triggered peripheral pixels are cropped and not considered. Figure 19 shows the median intensity of each frame of lidar data (after cropping) as a function of frame number for an ascent/descent maneuver at the six degree FOV setting. Figure 20 shows the accompanying median range for each frame of lidar data (after cropping) as a function of frame number. Due to the cropping process, maximum range is not defined as the point where $90 \%$ of the pixels trigger. Maximum range is identified for the var-FOV lidar through comparison of Figures 19 and 20. Figure 20 starts by showing a range value close to $500 \mathrm{~m}$, then the range spikes upward discontinuously to $1100 \mathrm{~m}$ and then returns discontinuously back to $500 \mathrm{~m}$. The discontinuous change is likely the result of a banking maneuver of the helicopter performed to set up for the run which momentarily resulted in the lidar being pointed at a distant area during the turn. The ascent maneuver begins, after a short descent to complete the setup for the run, and peaks out at $1200 \mathrm{~m}$ at which point a descent begins to setup for the next run. The Figure 19 median intensity trends support the same profile of setup and then ascent to a maximum.

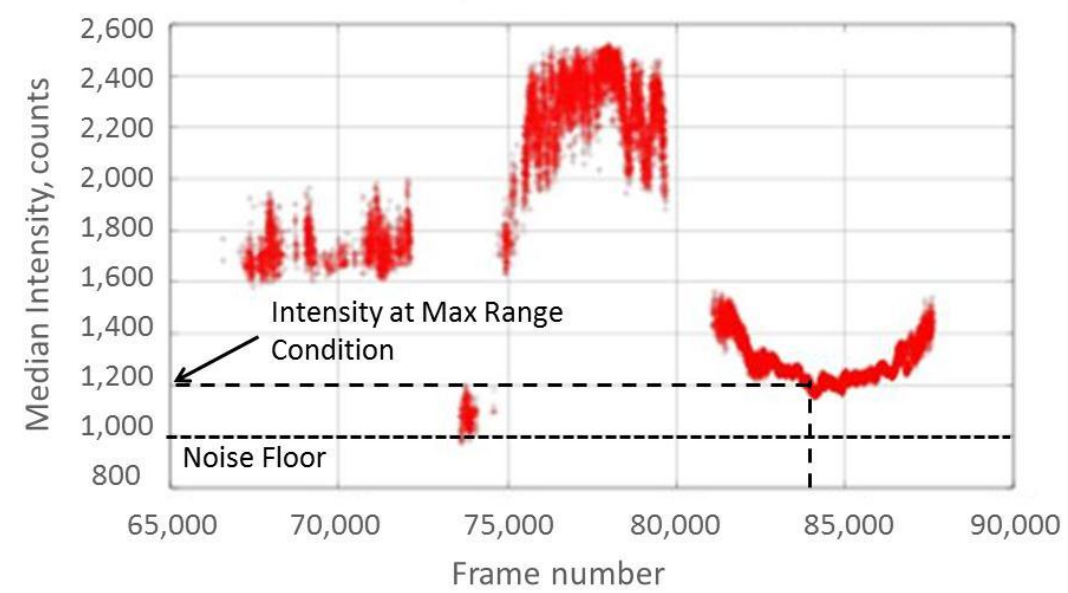

Figure 19: Flight \#5 (var-FOV lidar) ascent/descent maneuver median intensity of all triggered pixels in the cropped region as a function of frame number ( 30 frames / sec) acquired in flight at the six degree FOV setting with the noise floor at approximately 1000 counts. All data resides in the linear range. Intensity at maximum range is approximately 1200 counts at frame number $83,500$. 


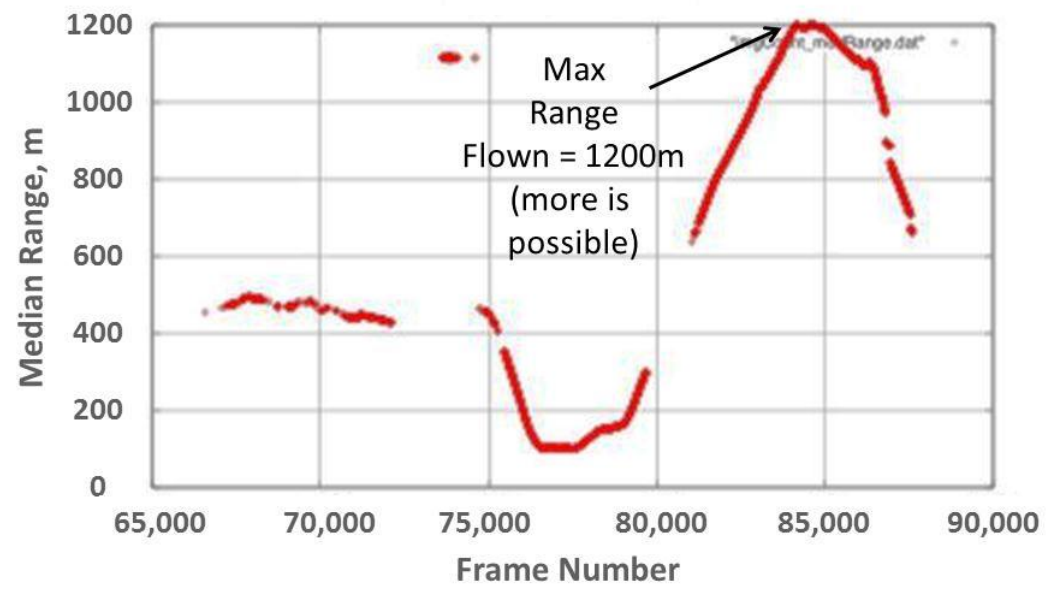

Figure 20: Flight \#5 (var-FOV lidar) ascent/descent maneuver median range of all triggered pixels as a function of frame number (30 frames / sec) at the six degree FOV setting. Maximum range is shown to be approximately $1200 \mathrm{~m}$ at frame number 83,500.

The var-FOV lab characterization and flight results for maximum range are in reasonable agreement based the setup differences between the lab and flight environments. The lab results indicate a maximum operational range of $1,600 \mathrm{~m}$ while the flight results indicate approximately $1,200 \mathrm{~m}$. In flight, additional slant range could have been achieved since the average intensity was shown to be approximately 200 counts above the noise floor. The sand reflectivity at $1.06 \mu \mathrm{m}$ is $40 \%$ at the $11^{\circ}$ incidence angle at which the $1200 \mathrm{~m}$ data is acquired, while the lab characterization target board reflectivity is $46 \%$ at its $0^{\circ}$ incidence angle which would artificially elevate the lab results.

\section{FLASH LIDAR TECHNOLOGY ADVANCEMENT}

The present development and field test cycle has taken the flash lidar subsystem technology one step closer to achieving the long-range ALHAT goals en-route to attaining a TRL of 6 with the full, integrated ALHAT system. Table 3 compares the achievements from the present development and test effort to the long-range ALHAT goals. The maximum operational range achievement shown is that of the var-FOV lidar since it incorporates the Fibertek laser which is the laser planned for the final ALHAT system. A 128 x 128 pixel array camera was successfully demonstrated, however, a factor of four additional pixels will ultimately be needed in order to meet the spatial precision requirements for hazard detection at maximum range with larger FOV's. The FOV needs to be extended from $16^{\circ}$ to $24^{\circ}$ to meet the long-range goals. The shortfall arose from a failure by the optics vendor to deliver a working mono-static transmitter optic system (i.e. coaxial optics used by both the receiver and the transmitter) and the resulting bi-static in-house system developed on short notice. Only the $6^{\circ} \mathrm{FOV}$ case was demonstrated in flight. The frame rate long-range goal has been achieved. The long-range goal for real-time application of range / intensity calibration coefficients has been achieved.

Table 3: Summary of achievements from present development / test cycle compared to ALHAT long-range goals.

\begin{tabular}{|c||c||c|}
\hline Parameter & $\begin{array}{c}\text { Field Test 4 } \\
\text { Achievements }\end{array}$ & $\begin{array}{c}\text { ALHAT } \\
\text { Long-Range } \\
\text { Goals }\end{array}$ \\
\hline Max operational range & $1200 \mathrm{~m}$ & $>1000 \mathrm{~m}$ \\
\hline \# pixels & $128 \times 128$ & $256 \times 256$ \\
\hline FoV & $1^{\circ},{ }^{*} 6^{\circ}$ to $16^{\circ}$ & $\begin{array}{c}\text { Variable } 6^{\circ} \\
\text { to } 24^{\circ}\end{array}$ \\
\hline Frame Rate & $30 \mathrm{~Hz}$ & $30 \mathrm{~Hz}$ \\
\hline Range / Intensity Calibration Application & Real-time & Real-time \\
\hline * In-flight demonstration limited to $6^{\circ}$ & &
\end{tabular}

*In-flight demonstration limited to $6^{\circ}$ 
Sensor technology upgrades are needed in order to close the gap between the present state and the long-range goals to ready the systems and hazard detection algorithms for use on a space mission. Higher sensitivity camera FPA's are being pursued which incorporate a more sensitive detector array as well as a lower noise ROIC which should lower the noise floor (i.e. increase dynamic range) and allow camera operations at lower detection threshold levels to observe fainter signals from farther ranges. An alternative 3-D imaging camera is being tested which approaches range detection in an analog method, versus the present camera's digital method which has the promise of increased sensitivity and dynamic range. Improved spatial precision is being pursued through prototype detectors and ROICs with a factor of four more pixels to permit hazard detection at maximum range with a larger FOV. Spatial precision improvement is also being pursued via a real-time image enhancement technique referred to as Super Resolution which is similar to the familiar digital magnification function of consumer cameras. The super resolution technique effectively increases the number of pixels as much as a factor of 8 by combining successive images, each of which are shifted in space by either vehicle motion or vibration, in a way in which information between physical pixels is filled in. Improvements are being pursued in zoom optics both for the receiver zoom as well as for the transmitter zoom to enable realization of the full $6^{\circ}$ to $24^{\circ}$ travel as well as miniaturization of the system flown in the present field test series.

\section{CONCLUSIONS}

Flash lidar technology is being evaluated by NASA to serve as the key hazard detection sensor in the GN\&C system for future robotic and crewed landers because of its ability to generate 3-D images of the potential landing site in real-time for use in identifying hazardous craters, rocks and sloped terrain. Future missions will send landers to increasing complex sites (poorly lit, challenging terrain, etc.) on differing planetary bodies (moon, Mars, asteroids) due to the high scientific and mission payoff possible and will require them to do so with higher fidelity and safety. Two flash lidars, integrated from a number of cutting-edge components from industry and NASA, have been lab characterized and flight tested for maximum operational range under the ALHAT project (in its fourth development and field test cycle) which is seeking to develop a GN\&C and sensing system based on lidar technology to carry out such missions. The flash lidars incorporate pioneering 3-D imaging cameras based on InGaAs APD and novel micro-electronic technology, high pulseenergy $1.06 \mu \mathrm{m} \mathrm{Nd:YAG} \mathrm{lasers,} \mathrm{and} \mathrm{high} \mathrm{performance} \mathrm{transmitter} \mathrm{and} \mathrm{receiver} \mathrm{fixed} \mathrm{and} \mathrm{zoom} \mathrm{optics.} \mathrm{The} \mathrm{two} \mathrm{flash}$ lidars (a fixed-FOV for use in a mosaic scanning technique and a var-FOV for use in a staring technique) were characterized on the LaRC Sensor Test Range, integrated with other portions of the ALHAT GN\&C system from partner organizations into an instrument pod at NASA-JPL, integrated onto an Erickson Aircrane Helicopter at NASA-Dryden, and flight tested at the Edwards AFB's Rogers dry lakebed over a field of geometric hazards during the summer of 2010. The maximum range goal of $1 \mathrm{~km}$ was achieved and exceeded up to a slant range of $1.2 \mathrm{~km}$. In addition, calibrated 3-D images of several hazards were acquired in real-time for later reconstruction into DEM's. A portion of the final ALHAT FOV goal was achieved. The tests helped to identify key technology advancement areas, to provide data on which to exercise signal processing and hazard detection algorithms, and to move the ALHAT system one step closer to its TRL 6 goal.

\section{ACKNOWLEDGEMENTS}

The authors would like to express appreciation to the ALHAT project manager, Chirold Epp of the NASA-Johnson Space Center, for his invaluable support. The authors would like to thank the NASA-Jet Propulsion Laboratory ALHAT team for facilitating the aircraft flight test campaign. The authors would like to acknowledge Andrew Johnson for his generation of the median range and median intensity plots from Flight \#5. The authors are also thankful to Advanced Scientific Concepts, Inc. for providing the flash lidar cameras and for supporting the static and airborne tests.

\section{REFERENCES}

[1] Brady, T., and Paschall, S., "The Challenge of Safe Lunar Landing," Proc. of IEEE Aerospace Conference, Big Sky, MT, 1 - 14 (2010).

[2] Epp, C.D., Robinson, E.A., and Brady, T., "Autonomous Landing and Hazard Avoidance Technology (ALHAT)”, Proc. IEEE Aerospace Conference, 1-7 (2008). 
[3] Amzajerdian, F., Pierrottet, D., Tolson, R. H., Powell, R. W., John B. Davidson, and Peri, F., "Development of a Coherent Lidar for Aiding Precision Soft Landing on Planetary Bodies," Proc. of $13^{\text {th }}$ Coherent Laser Radar Conference, Kamakura, Japan (2005).

[4] Pierrottet, D., Amzajerdian, F., Petway, L., Barnes, B., and Lockard, G., "Flight test performance of a high precision navigation Doppler lidar," Proc. SPIE 7323, (2009).

[5] Amzajerdian, F., Vanek, M., Petway, L., Pierrottet, D., Busch, G., and Bulyshev, A., "Utilization of 3-D Imaging Flash Lidar Technology for Autonomous Safe Landing on Planetary Bodies,” Proc. SPIE 7608, (2010).

[6] Stettner, R., “Compact 3-D Flash LIDAR Video Cameras and Applications,” Proc. SPIE 7684, (2010).

[7] Wolfe, W. L. and Zissis, G. J., [The Infrared Handbook], Infrared Information and Analysis (IRIA) Center, Michigan, 23-6 (1985).

[8] Pierrottet, D., Amzajerdian, F., Meadows, B., Estes, R., Noe, A., "Characterization of 3-D imaging lidar for hazard avoidance and autonomous landing on the Moon," Proc. SPIE 6550, (2007). 